\title{
Kinetics of $3^{\prime}$-Deoxy-3'-18 F-Fluorothymidine During Treatment Monitoring of Recurrent High-Grade Glioma
}

\author{
Christiaan Schiepers ${ }^{1}$, Magnus Dahlbom ${ }^{1}$, Wei Chen ${ }^{1}$, Timothy Cloughesy ${ }^{2}$, Johannes Czernin ${ }^{1}$, \\ Michael E. Phelps ${ }^{1}$, and Sung-Cheng Huang ${ }^{1}$ \\ ${ }^{I}$ Department of Molecular and Medical Pharmacology, David Geffen School of Medicine, University of California, Los Angeles, \\ California; and ${ }^{2}$ Department of Neurology, David Geffen School of Medicine, University of California, Los Angeles, California
}

$3^{\prime}$-deoxy-3'-18 F-fluorothymidine ( $\left.{ }^{18} \mathrm{~F}-\mathrm{FLT}\right)$ is used as a biomarker of cell proliferation. We investigated the kinetics of ${ }^{18} \mathrm{~F}-\mathrm{FLT}$ during treatment of malignant glioma with bevacizumab and irinotecan. Methods: Fifteen patients with recurrent high-grade brain tumors (2 grade III, 13 grade IV) were studied at baseline (study 1 [S1]), after 1 course of therapy (2 wk, study 2 [S2]), and at the end of therapy (6 wk, study 3 [S3]). ${ }^{18} \mathrm{~F}-\mathrm{FLT}(1.5 \mathrm{MBq} / \mathrm{kg}$ ) was administered intravenously, and dynamic PET was performed for $1 \mathrm{~h}$. Curves representing blood clearance and tumor uptake were derived from factor images and summed frames with thresholding techniques or with a fixed cube. The standard ${ }^{18} \mathrm{~F}-\mathrm{FLT}$ model was used to estimate the rate constants. ${ }^{18} \mathrm{~F}$ FLT uptake was measured at 2 time points (early standardized uptake value [SUV early $]$ and late SUV [SUV late $\left._{\text {la }}\right]$. Results: Parameters appeared similar for curves derived from factor images and summed frames; the steepest drop occurred between S1 and S2 for transport, influx, SUV early, and SUV late. Three groups were distinguished on the basis of clinical outcome: patients who died within 6 mo (group 1 [G1], $n=4$ ), survived 6-12 mo (group 2 [G2], $n=6$ ), and survived more than 1 y (group 3 [G3], $n=5$ ). None of the rate constants was significantly different between the groups. Long-term survivors (G3) showed a significantly different SUV change (in percentage) between S1 and S3, whereas short-term survivors (G1 and G2) did not. Conclusion: Overall, the relative SUV change from $S 1$ to $S 3$ predicted a favorable clinical outcome, whereas the SUV change from S1 to S2 did not. Long-term survivors (G3) showed a significant drop in SUV from $S 1$ to $S 2$ and from S1 to S3. Significant correlations were found between SUV and both the rate constant and the influx rate. The correlation coefficient between $S_{U} V_{\text {late }}$ and influx rate was 0.91 , permitting response monitoring by the measurement of ${ }^{18} \mathrm{~F}-\mathrm{FLT}$ uptake changes.

Key Words: ${ }^{18} \mathrm{~F}-\mathrm{FLT}$ (3'-deoxy-3'-18F-fluorothymidine); positron emission tomography; malignant brain tumor; kinetic modeling; factor analysis; therapy monitoring

J Nucl Med 2010; 51:720-727

DOI: 10.2967/jnumed.109.068361

Received Jul. 13, 2009; revision accepted Jan. 26, 2010.

For correspondence or reprints contact: Christiaan Schiepers, Molecular and Medical Pharmacology, David Geffen School of Medicine, MP200B114, 10833 Le Conte Ave., Los Angeles, CA 90095-6942.

E-mail: cschiepers@mednet.ucla.edu

Guest Editor: Wolf-Dieter Heiss, Max-Planck-Institut für Neurologische Forschung

COPYRIGHT $\odot 2010$ by the Society of Nuclear Medicine, Inc.
$\mathbf{T}$ he imaging of cell proliferation has emerged as an avenue of monitoring neoplastic disease. 3'-deoxy-3'fluorothymidine (FLT) labeled with ${ }^{18} \mathrm{~F}$ has provided a noninvasive way of studying cell proliferation. Although ${ }^{18} \mathrm{~F}-\mathrm{FLT}$ is not incorporated into DNA, it is phosphorylated by thymidine kinase-1 and trapped inside the cytosol. Phosphorylated ${ }^{18} \mathrm{~F}$-FLT appears suitable for imaging with PET $(1,2)$ and represents thymidine incorporation into DNA, as has been demonstrated in various tumor models including 2 glioma cell lines (3). The clinical application of ${ }^{18} \mathrm{~F}-\mathrm{FLT}$ in brain gliomas has been reported $(4,5)$. The kinetic model has been described elsewhere (6).

Various tracers have been used to monitor therapy. For PET and glioma, results have been reported for ${ }^{18} \mathrm{~F}-\mathrm{FDG}$, ${ }^{11} \mathrm{C}$-methionine, ${ }^{18} \mathrm{~F}$-tyrosine, and others $(7-11)$.

The National Cancer Institute trial on recurrent glioma using PET and ${ }^{18}$ F-FLT showed that the influx rate $K_{i}$ and phosphorylation rate $k_{3}$ reached statistical significance in separating recurrent glioma $(n=15)$ from radiation necrosis $(n=4)(12)$. It also reported that estimates of the standardized uptake value (SUV) or visual analysis were not able to distinguish these entities. In addition, the authors of this report found that ${ }^{18} \mathrm{~F}-\mathrm{FLT}$ performed better than ${ }^{18} \mathrm{~F}$-FDG.

In a previous study (5), the percentage change in ${ }^{18} \mathrm{~F}-\mathrm{FLT}$ uptake was used to categorize patients as responders (SUV decrease $>25 \%$ ) or nonresponders. In the current study, we investigated the kinetics of ${ }^{18} \mathrm{~F}$-FLT of recurrent high-grade brain tumors during treatment. These parameters provide more information than did the ${ }^{18}$ F-FLT uptake alone, and our hypothesis is that they contain predictive information. In addition, we evaluated the effect of different analysis methods and compared their results. A volume of interest (VOI) was defined to generate the input curve and another to derive the output curve. Two types of VOI were used of variable or fixed size and defined on parametric or composite images. These methods can be automated easily and are suitable quantification techniques for routine clinical applications. 
The tracer kinetics were investigated with compartmental modeling. In this study, the absolute value of parameters, and their relative change, was evaluated, and the different analysis techniques were compared. In addition, ${ }^{18} \mathrm{~F}$-FLT uptake was measured at early and late time points and expressed as SUV. The final results were correlated to clinical outcome and survival.

\section{MATERIALS AND METHODS}

\section{Patients}

Twenty patients with recurrent glioma were prospectively enrolled. Nine were included from our previous study (5), and 11 were newly enrolled. All patients underwent dynamic ${ }^{18} \mathrm{~F}$-FLT studies at baseline and during therapy, after which, 4 patients had to be removed because of technical issues ( 2 had severe head motion during the study, 1 had an incomplete study, and the other had an infiltrated dose [inadequate bolus with activity remaining in the arm]). One patient had a complete response to therapy; no tumor could be delineated in the ${ }^{18}$ F-FLT PET study after treatment, and kinetic modeling failed.

The final study population consisted of 15 patients (6 men and 9 women; average age, $53 \mathrm{y}$; age range, 26-77 y). The study was approved by our Institutional Review Board, and all patients signed an informed consent form. Histopathology revealed 13 grade IV tumors (glioblastoma multiforme) and 2 grade III tumors (anaplastic astrocytoma and anaplastic mixed glioma). Each patient underwent 3 studies: at baseline (study 1 [S1]), after 1 course of therapy (average interval, $16 \pm 6 \mathrm{~d}$; study 2 [S2]), and after 3 courses of therapy (average interval, $30 \pm 8 \mathrm{~d}$; study 3 [S3]). For every patient, the most active tumor (i.e., highest ${ }^{18} \mathrm{~F}-$ FLT uptake) at baseline was selected. Therapy consisted of biweekly cycles of bevacizumab $(10 \mathrm{mg} / \mathrm{kg})$ and irinotecan $(125$ or $350 \mathrm{mg} / \mathrm{m}^{2}$ ). Table 1 shows the population data, grade, number of treatments, and pathology of the malignant gliomas.

\section{Image Acquisition}

${ }^{18} \mathrm{~F}$-FLT was synthesized by a modification of a previously reported procedure (13). A $1.5 \mathrm{MBq} / \mathrm{kg}(0.04 \mathrm{mCi} / \mathrm{kg})$ dose of ${ }^{18}$ F-labeled FLT was administered. PET was performed using an ECAT HR+ system (CTI/Siemens). A transmission scan (2dimensional mode) of 5 -min duration was acquired first. ${ }^{18} \mathrm{~F}$ FLT was administered intravenously, and a dynamic acquisition (3-dimensional mode) was started simultaneously. Twenty-three frames $(8 \times 15,2 \times 30,2 \times 60$, and $11 \times 300 \mathrm{~s})$ were acquired. The images were reconstructed with iterative techniques as described elsewhere (14). The following parameters were used: maximum a posteriori for the transmission scan and ordered-subset expectation maximization for the emission scan, corrections for attenuation and scatter, and a gaussian kernel of $5 \mathrm{~mm}$ in full width at half maximum as a postreconstruction smoothing filter. The final reconstructed volume set had a matrix size of $128 \times 128$ and consisted of 63 planes. The voxel size was $2.06 \times 2.06 \times 2.425 \mathrm{~mm}$, using a zoom of 2.5 in the axial planes.

\section{Processing}

Factor analysis was performed on the volume set of reconstructed images as described elsewhere (15). Briefly, factor analysis evaluates temporal changes in dynamic datasets and generates functional images of the vessels, tumor, brain structures, bone marrow, and the like. Rebinning produced a dataset with $4.1 \times 4.1 \times 4.9 \mathrm{~mm}$ voxels that was used for further processing. For our application, factor analysis generated 3 factors and corresponding factor images, which are the associated spatial distribution of a factor. Factor 1 represents the large vessels and factor 2 the tumors in the brain. For every patient, studies were available at 3 time points (S1, S2, and S3). For the blood curve, VOIs were generated using a $50 \%$ threshold of the maximum voxel in the transverse sinuses. These VOIs for the input function were generated completely automatically with our processing modules. For the brain tumor, VOIs were derived from factor images (F2) or summation of frames between 45 and $60 \mathrm{~min}$

\begin{tabular}{|c|c|c|c|c|c|c|c|c|c|c|c|}
\hline Group & Patient & Sex & $\begin{array}{l}\text { Age } \\
\text { (y) }\end{array}$ & $\begin{array}{c}\text { Initial } \\
\text { pathology }\end{array}$ & $\begin{array}{c}\text { World Health } \\
\text { Organization } \\
\text { grade }\end{array}$ & Initial therapy & $\begin{array}{l}\text { Interval } \\
\text { (d) }\end{array}$ & $\begin{array}{l}\text { Pathology } \\
\text { recurrence }\end{array}$ & $\begin{array}{c}\text { World Health } \\
\text { Organization grade } \\
\text { recurrence }\end{array}$ & $\begin{array}{c}\text { Prior } \\
\text { treatments }\end{array}$ & $\begin{array}{l}\text { Surviva } \\
\text { (d) }\end{array}$ \\
\hline \multirow[t]{4}{*}{1} & 1 & M & 69 & GBM & 4 & Chemotherapy/XRT & 76 & GBM & 4 & 1 & 81 \\
\hline & 2 & $\mathrm{~F}$ & 65 & GBM & 4 & Chemotherapy/XRT & 578 & GBM & 4 & 2 & 107 \\
\hline & 3 & M & 50 & AA & 3 & Chemotherapy/XRT & 332 & AA & 3 & 2 & 59 \\
\hline & 4 & $\mathrm{~F}$ & 59 & GBM & 4 & Chemotherapy/XRT & 322 & GBM & 4 & 1 & 169 \\
\hline \multirow[t]{6}{*}{2} & 5 & $\mathrm{~F}$ & 37 & AMG & 3 & Chemotherapy/XRT & 160 & AMG & 3 & 1 & 260 \\
\hline & 6 & M & 64 & GBM & 4 & Chemotherapy/XRT & 178 & GBM & 4 & 1 & 234 \\
\hline & 7 & M & 26 & $\mathrm{AA}$ & 3 & Chemotherapy & 462 & GBM & 4 & 3 & 364 \\
\hline & 8 & $\mathrm{~F}$ & 35 & $\mathrm{AA}$ & 3 & XRT & 2,804 & GBM & 4 & 3 & 318 \\
\hline & 9 & M & 45 & GBM & 4 & Chemotherapy/XRT & 132 & GBM & 4 & 1 & 344 \\
\hline & 10 & $\mathrm{~F}$ & 54 & GBM & 4 & Chemotherapy/XRT & 91 & GBM & 4 & 1 & 343 \\
\hline \multirow[t]{5}{*}{3} & 11 & $\mathrm{~F}$ & 61 & GBM & 4 & Chemotherapy/XRT & 719 & GBM & 4 & 2 & 400 \\
\hline & 12 & $\mathrm{~F}$ & 62 & GBM & 4 & Chemotherapy/XRT & 316 & GBM & 4 & 1 & 709 \\
\hline & 13 & $\mathrm{~F}$ & 47 & GBM & 4 & Chemotherapy/XRT & 160 & GBM & 4 & 1 & 374 \\
\hline & 14 & M & 76 & GBM & 4 & Chemotherapy/XRT & 134 & GBM & 4 & 1 & 978 \\
\hline & 15 & $\mathrm{~F}$ & 40 & GBM & 4 & Chemotherapy/XRT & 462 & GBM & 4 & 1 & 366 \\
\hline
\end{tabular}


(Sum), using simple thresholding techniques. The effect of varying isocontours $(55 \%-75 \%)$ was investigated. In this threshold method, both the shape and the size of the VOI varied between studies. In another analysis, a cube of $5 \times 5 \times 5$ voxels $(10 \times 10 \times$ $12 \mathrm{~mm}=1.2 \mathrm{~cm}^{3}$ ) was generated and positioned around the voxel with maximum tumor uptake on the factor images (F2-cube) or summed frames (Sum-cube). In this cube analysis, the shape of the VOI is constant across studies, but the location is related to the maximum count in the tumor.

\section{Compartmental Model}

The kinetic model for ${ }^{18} \mathrm{~F}$-FLT is based on the original work of Shields et al. (1). Our implementation for the brain was published elsewhere (6). Briefly, the model assumes a transport step of ${ }^{18} \mathrm{~F}$ FLT from the vascular to the tissue space. There is no partitioning in the vascular space, and plasma and whole-blood activity concentrations are the same (16). In the tissue space, there is an exchangeable compartment (with intact ${ }^{18} \mathrm{~F}-\mathrm{FLT}$ ) and a compartment with phosphorylated ${ }^{18} \mathrm{~F}-\mathrm{FLT}$ (mono-, di-, and triphosphate $\left.{ }^{18} \mathrm{~F}-\mathrm{FLT}\right) .{ }^{18} \mathrm{~F}-\mathrm{FLT}$ is metabolized by the liver to ${ }^{18} \mathrm{~F}-\mathrm{FLT}-$ glucoronide, which is present only in the vascular space-that is, not transported into the brain-tissue compartment. Metabolites were corrected by subtracting a metabolite fraction from the vascular curve. This fraction was calculated as $0.42 \times(1-$ $\exp [-0.029 \times$ time $])$ as explained in our earlier publication $(6)$. Kinetic modeling yields the 4 rate constants $\left(k_{1}, k_{2}, k_{3}\right.$, and $\left.k_{4}\right)$ between compartments. The blood volume fraction in tissue $V_{b}$ was estimated as a fifth parameter. The influx rate constant $K_{i}$ was calculated as $k_{1} k_{3}\left(k_{2}+k_{3}\right)^{-1}$ and the volume of distribution $\mathrm{V}_{\mathrm{d}}$ as $k_{1}\left(k_{2}+k_{3}\right)^{-1}$. Metabolite correction of the blood curve to yield the input function was performed as described previously (6). A recovery coefficient of 0.7 was chosen to convert the image-based blood time-activity curve to the input function. Because the tumors, compared with the image resolution, are relatively large the recovery coefficient of the output function was set to unity.

\section{Outcome Analysis}

Of the 15 patients studied, 4 died within 4 mo (group 1 [G1]) and 6 died between 6 and 12 mo (group 2 [G2]). The remaining 5 survived for more than 1 y (group 3 [G3]). The overall survival time and time between initial diagnosis and current study treatment are given in Table 1. The median survival was $94 \mathrm{~d}$ for G1, $331 \mathrm{~d}$ for G2, and $542 \mathrm{~d}$ for G3. The patient with the complete response (no ${ }^{18} \mathrm{~F}-\mathrm{FLT}$ uptake after treatment) had an overall survival of $1,054 \mathrm{~d}$.

\section{Statistical Analysis}

Results are presented as the mean $\pm \mathrm{SD}$. The Student $t$ test was used to compare the kinetic parameters and ${ }^{18} \mathrm{~F}$-FLT uptake. For small sample sizes $(n<10)$, the Wilcoxon Mann-Whitney $U$ test (2-sample rank test) was applied. Pearson correlation was used to study the relationship between subgroups.

\section{RESULTS}

In Figure 1, for each of the 3 studies a representative factor slice is shown for a short-term survivor (G1). Figure 2 shows an example for a long-term survivor (G3).

Comparison of the varying thresholds of the tumor VOI delineated on the factor images ( $\mathrm{F} 2$, threshold $55 \%-75 \%$ ) showed similar results for the parameter values of $k_{1}, \mathrm{~K}_{\mathrm{i}}$, $\mathrm{V}_{\mathrm{b}}$, early SUV $\left(\mathrm{SUV}_{\text {early }}\right)$, and late $\mathrm{SUV}\left(\mathrm{SUV}_{\text {late }}\right)$. The correlation coefficients of these parameters between thresholding methods were high and ranged from 0.91 to 0.99 both for the entire set $(n=45)$ and for the 3 study sets (S1, $\mathrm{S} 2$, and S3; $n=15$ each). Intermediate correlations $(0.7<$ $r<0.9)$ were found for $k_{2}$ and $\mathrm{V}_{\mathrm{d}}$, whereas $k_{3}$ and $k_{4}$ showed poor correlations $(r<0.6)$. Figure 3 shows the average results of all patients for the 3 sets of studies, using tumor VOIs delineated on summed frames (Sum-75\%). Results in Figure 3 suggest a trend for $k_{1}$, but no significantly different results were obtained for the absolute value of the parameters tested. The study after the first course of treatment (S2) generally shows lower parameter values, with a tendency to rebound at S3, except for $k_{4}$. Considering the absolute values of the entire population, no statistical differences were found for the micro- or macroparameters between time points $\mathrm{S} 1, \mathrm{~S} 2$, and $\mathrm{S} 3$ for any of the analysis methods (F2-55\%-75\%, Sum-75\%, F2-cube, or Sum-cube).

Subsequently, the relative parameter change defined as $(\mathrm{S} 1-\mathrm{S} 2) / \mathrm{S} 1$ or $(\mathrm{S} 1-\mathrm{S} 3) / \mathrm{S} 1$ (i.e., fraction of change) was evaluated. In Figure 4, box plots are shown depicting the relative changes between $\mathrm{S} 1$ and $\mathrm{S} 2$ (left panel) and between S1 and S3 (right panel). Figure 4 shows narrow plots for $k_{1}$ at an early time point, with large variability later for G1 and G2. G3, on the other hand, shows consistently small variability between $\mathrm{S} 1$ and $\mathrm{S} 3$ for all 3 parameters. The variability of $k_{3}$ is large (except of G3 for $\mathrm{S} 1 \rightarrow \mathrm{S} 3$ ), signifying large fluctuations in phosphorylation rate during therapy.

Table 2 shows the population mean using the factor images with varying threshold and the summed images with $75 \%$ threshold. Inspection of the cube VOIs showed a similar pattern. None of the parameter values showed significant differences between the 4 methods analyzed:

FIGURE 1. Representative factor images from patient 2 of G1. Note increasing intensity (proportional to ${ }^{18} \mathrm{~F}-\mathrm{FLT}$ influx rate) from S1 to S3. Images are displayed using same color scale (shown in arbitrary units).

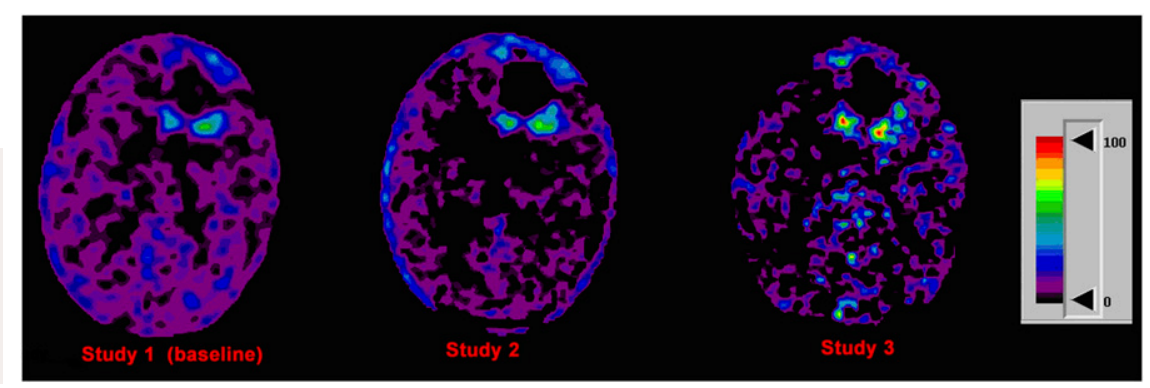




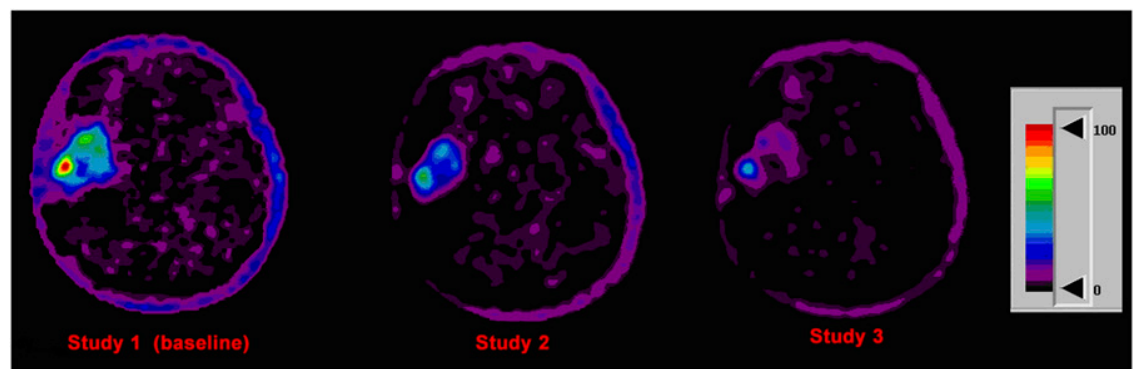

FIGURE 2. Representative factor images from patient 13 of G3. Note decreasing ${ }^{18} \mathrm{~F}-\mathrm{FLT}$ influx rate from $\mathrm{S} 1$ to S3. Images are displayed using same color scale (in arbitrary units).

threshold VOI or cube VOI on factor or summed images. When we compared the tumor volume (in voxels), VOIs obtained with a threshold of $70 \%$ on the factor images (F2-70\%) fitted best with a threshold of $75 \%$ on the summed images (Sum-75\%), and linear regression analysis furnished a slope of 1.050 and intercept of $0.077(r=0.87)$. Figure 5 shows the scatter diagram for $\mathrm{K}_{\mathrm{i}}$ for a variable VOI (F2-70\%) versus a fixed VOI (Sum-cube).

The same techniques were used to measure the SUV, either threshold VOIs or a fixed cube, on the factor or summed images. ${ }^{18} \mathrm{~F}$-FLT uptake reaches a maximum around 10-20 $\mathrm{min}(4,6)$; therefore, we calculated SUV early (from 10 to $25 \mathrm{~min}$ ) and $\mathrm{SUV}_{\text {late }}$ (from 50 to $60 \mathrm{~min}$ ). The average value for $\mathrm{SUV}_{\text {early }}$ was 0.83 and for $\mathrm{SUV}_{\text {late }} 0.85$. Both have a high correlation coefficient, compared with $\mathrm{K}_{\mathrm{i}}(0.86$ and 0.90 , respectively).

A scatter plot of $\mathrm{SUV}_{\text {late }}$ versus $\mathrm{K}_{\mathrm{i}}$ for the 3 studies is shown in Figure 6. The parameters at each time point, S1-

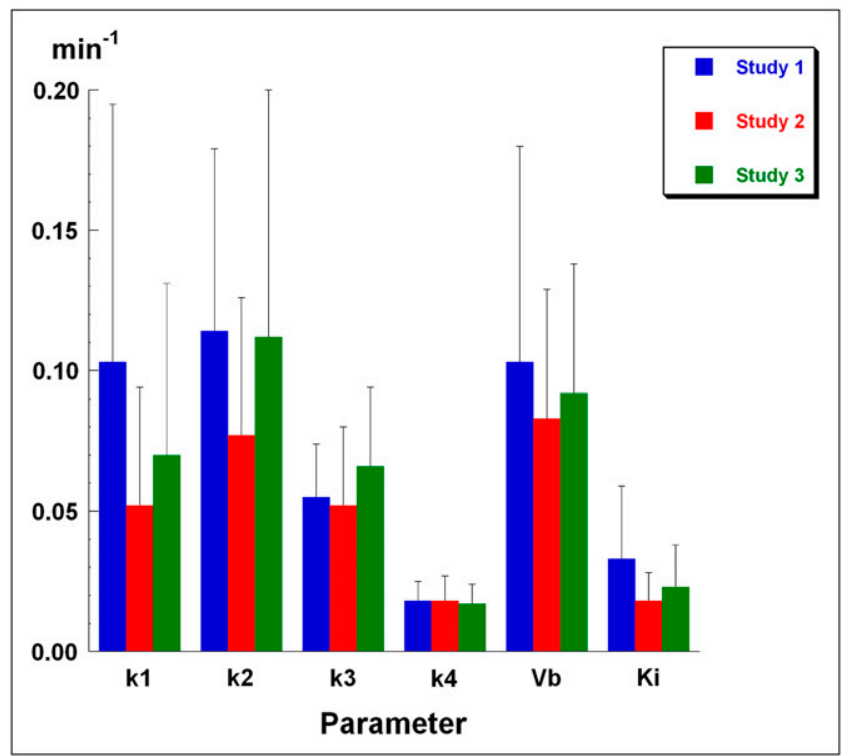

FIGURE 3. Average results per study (S1-S3) for rate constants $\left(k_{1}-k_{4}\right), \mathrm{V}_{\mathrm{b}}$, and $\mathrm{K}_{\mathrm{i}}$. Bars denote SD. VOls are delineated with threshold of $75 \%$ on summed images from 45 to $60 \min$. $n=15$ for each column. None of shown parameters demonstrates significant differences for mean between $\mathrm{S} 1, \mathrm{~S} 2$, or S3. Dimension of $k_{1}-k_{4}$ and $\mathrm{K}_{\mathrm{i}}$ is $\mathrm{min}^{-1}$, whereas $V_{b}$ is fraction (unitless).
S3, show an excellent correlation and similar slopes; none of the regression parameters was significantly different. The range of the parameters shows a decrease, both for $\mathrm{K}_{\mathrm{i}}$ and $\mathrm{SUV}_{\text {late, }}$, when proceeding from the baseline study $\mathrm{S} 1$ to either $\mathrm{S} 2$ or $\mathrm{S} 3$.

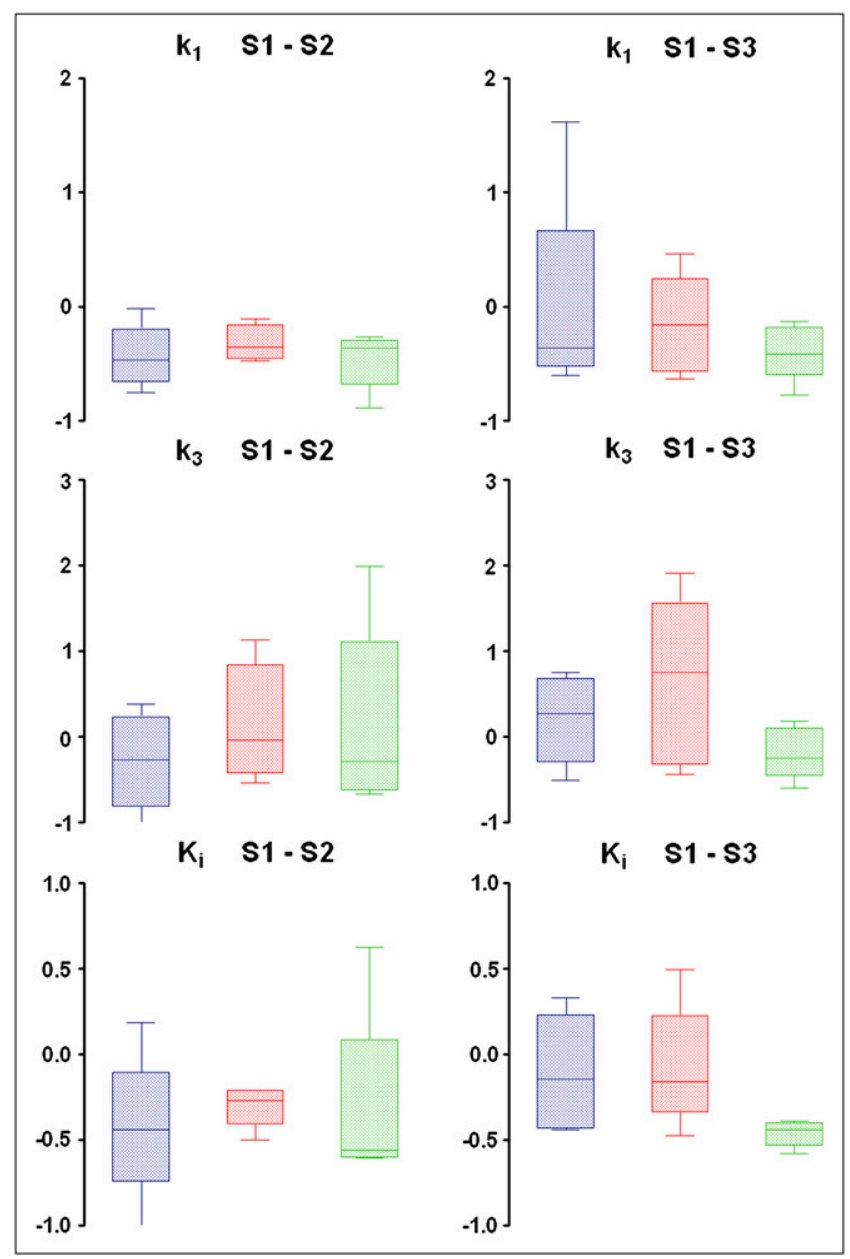

FIGURE 4. Box plots of relative changes for parameters $k_{1}$, $k_{3}$, and $\mathrm{K}_{\mathrm{i}}$. On left, relative changes between $\mathrm{S} 1$ and $\mathrm{S} 2$ are shown; on right, fractional changes between S1 and S3 are shown. Note small variability in $k_{1}$ for $\mathrm{S} 1 \rightarrow \mathrm{S} 2$ for all 3 groups. Variability for G3 (green) remains small for $\mathrm{S} 1 \rightarrow \mathrm{S} 3$ for all 3 parameters shown. For $k_{3}$, wide variability in relative changes exists, except for G3 between baseline and end of therapy. blue $=\mathrm{G} 1$; red $=\mathrm{G} 2$; green $=\mathrm{G} 3$. 

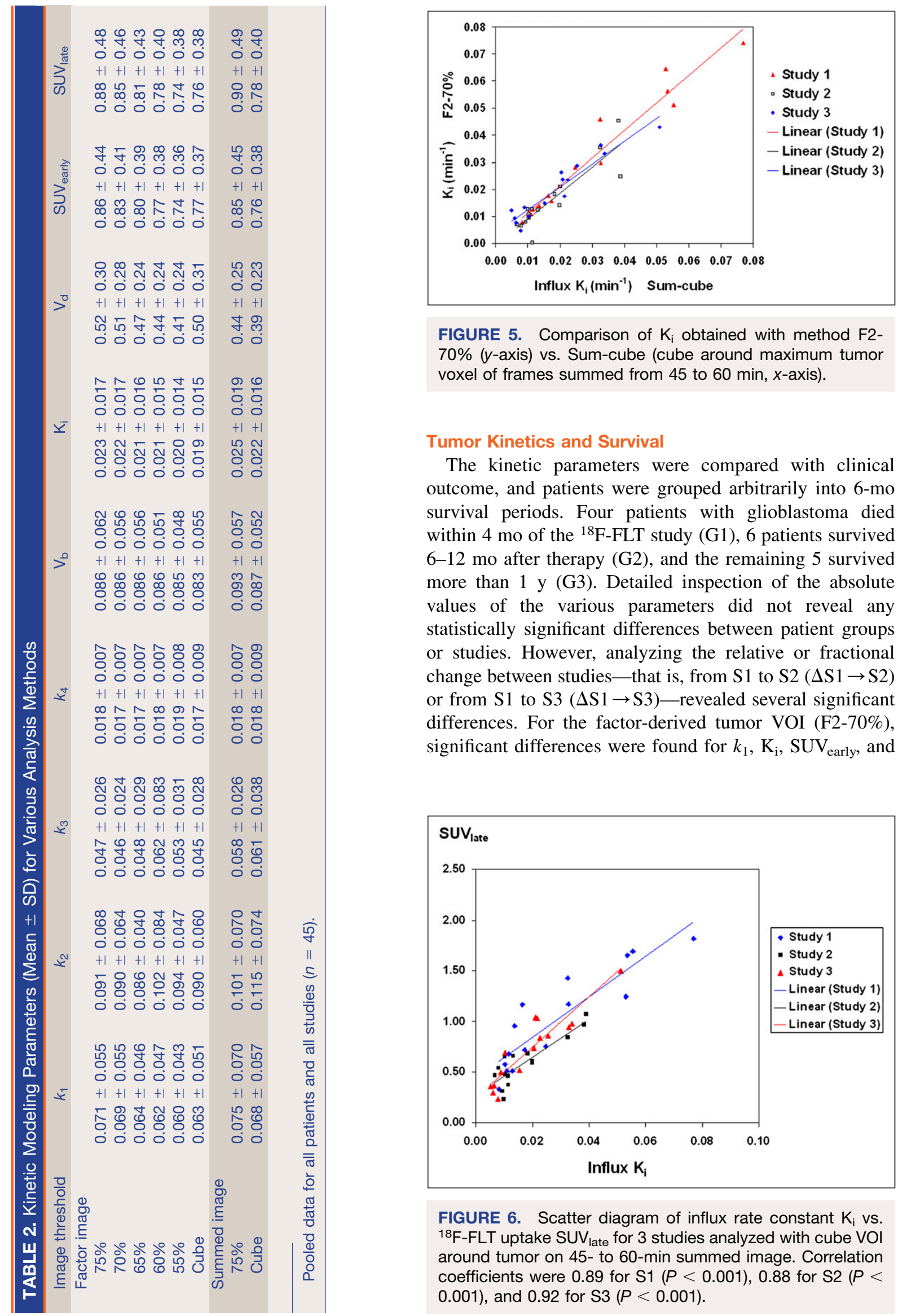

FIGURE 5. Comparison of $\mathrm{K}_{\mathrm{i}}$ obtained with method F2$70 \%$ ( $y$-axis) vs. Sum-cube (cube around maximum tumor voxel of frames summed from 45 to $60 \mathrm{~min}, x$-axis).

\section{Tumor Kinetics and Survival}

The kinetic parameters were compared with clinical outcome, and patients were grouped arbitrarily into 6-mo survival periods. Four patients with glioblastoma died within 4 mo of the ${ }^{18}$ F-FLT study (G1), 6 patients survived 6-12 mo after therapy (G2), and the remaining 5 survived more than 1 y (G3). Detailed inspection of the absolute values of the various parameters did not reveal any statistically significant differences between patient groups or studies. However, analyzing the relative or fractional change between studies- that is, from S1 to $\mathrm{S} 2(\Delta \mathrm{S} 1 \rightarrow \mathrm{S} 2)$ or from $\mathrm{S} 1$ to $\mathrm{S} 3(\Delta \mathrm{S} 1 \rightarrow \mathrm{S} 3)$-revealed several significant differences. For the factor-derived tumor VOI (F2-70\%), significant differences were found for $k_{1}, \mathrm{~K}_{\mathrm{i}}, \mathrm{SUV}_{\text {early }}$, and

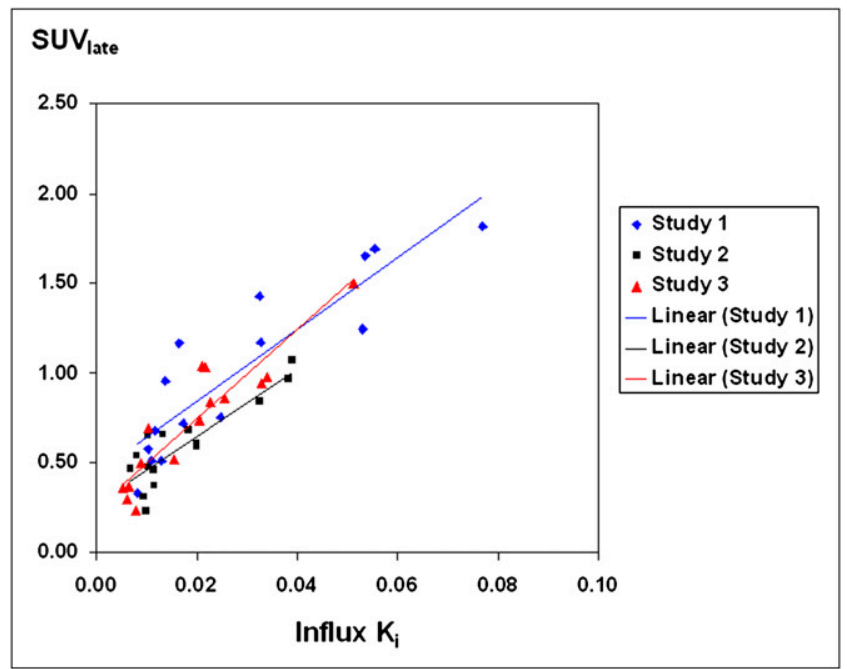

FIGURE 6. Scatter diagram of influx rate constant $\mathrm{K}_{\mathrm{i}}$ vs. ${ }^{18} \mathrm{~F}-\mathrm{FLT}$ uptake $\mathrm{SUV}_{\text {late }}$ for 3 studies analyzed with cube VOI around tumor on 45- to 60-min summed image. Correlation coefficients were 0.89 for S1 $(P<0.001), 0.88$ for S2 $(P<$ $0.001)$, and 0.92 for S3 $(P<0.001)$. 
$\mathrm{SUV}_{\text {late }}$ in G2 for $\Delta \mathrm{S} 1 \rightarrow \mathrm{S} 2$ and in G2 and G3 for both $\Delta \mathrm{S} 1 \rightarrow \mathrm{S} 2$ and $\Delta \mathrm{S} 1 \rightarrow \mathrm{S} 3$. For the Sum-75\%, tumor VOI significant differences in $\mathrm{K}_{\mathrm{i}}$ and SUV were found only for $\mathrm{G} 3$ for both $\Delta \mathrm{S} 1 \rightarrow \mathrm{S} 2$ and $\Delta \mathrm{S} 1 \rightarrow \mathrm{S} 3$. More significant differences were found for the cubes positioned around the maximum tumor uptake than for the variable VOIs. For F2cube, parameters $\mathrm{V}_{\mathrm{b}}, \mathrm{K}_{\mathrm{i}}, \mathrm{V}_{\mathrm{d}}$, and SUV showed a significant relative change in G3 for both $\Delta \mathrm{S} 1 \rightarrow \mathrm{S} 2$ and $\Delta \mathrm{S} 1 \rightarrow \mathrm{S} 3$. The same was true for Sum-cube. In addition, this latter cube VOI found significant differences in $\mathrm{G} 2$ for $\mathrm{K}_{\mathrm{i}}$ and $\mathrm{V}_{\mathrm{d}}$ both for $\Delta \mathrm{S} 1 \rightarrow \mathrm{S} 2$ and for $\Delta \mathrm{S} 1 \rightarrow \mathrm{S} 3$. Combining G1 and G2 $(n=10)$ and comparing these parameters with G3 $(n=$ 5) yielded the same statistically significant differences as before. Thus, most significant relative changes between studies were found with a cube of $5 \times 5 \times 5$ voxels around the tumor maximum. Using a variable tumor VOI reduced the number of significant changes, and in general, only relative changes in $\mathrm{K}_{\mathrm{i}}$ and SUV remained statistically significant. SUV changes were always significantly different for G3, both for early and for late time points (Fig. 7).

Results with regard to SUV changes of more than $25 \%$, which was the criterion used to define a responder in our earlier study (5), revealed that G3 had a 100\% sensitivity both for $\Delta \mathrm{S} 1 \rightarrow \mathrm{S} 2$ and for $\Delta \mathrm{S} 1 \rightarrow \mathrm{S} 3$. These relative changes were statistically significant $(P<0.01)$.

\section{DISCUSSION}

In this study, the kinetics of ${ }^{18} \mathrm{~F}-\mathrm{FLT}$ in malignant brain tumors were investigated at baseline and during treatment and stratified to clinical outcome. Our analysis is completely image-based and virtually operator-independent. The input function was derived automatically from factor images of the transverse sinuses; no user interaction was required. The tumor VOIs were derived with thresholding techniques or defined as a cube around the voxel with maximum ${ }^{18}$ F-FLT uptake. In earlier work, we have demonstrated that factor analysis can reliably generate input and output functions (17-22). In another study, we have shown that a general 3-compartment model is adequate for describing the ${ }^{18} \mathrm{~F}-\mathrm{FLT}$ kinetics of malignant brain tumors. As shown by Shields et al., the ${ }^{18}$ F-FLT model is a noninvasive way for evaluating cellular proliferation (2). ${ }^{18} \mathrm{~F}$-FLT influx rate is a representation of thymidine flux or incorporation rate into DNA (1), as was demonstrated by Chen et al. $(4,5)$ and by Ullrich et al., who found that ${ }^{18} \mathrm{~F}$-FLT uptake correlated with in vivo proliferation rate in newly diagnosed high-grade gliomas (11).

In this study, we used a total dynamic acquisition time of $1 \mathrm{~h}$. This acquisition time is $15 \mathrm{~min}$ shorter than that in our study of 2007 (16). The mathematic studies of Muzi et al. (16) have shown that the errors (defined as SEE/mean) increase when the acquisition duration is decreased from 120 to $60 \mathrm{~min}$. For $k_{1}$, the increase is from $13 \%$ to $15 \%$ and for $\mathrm{K}_{\mathrm{i}}$ from $4 \%$ to $9 \%$, which are still acceptable. The error for $k_{4}$ is $42 \%$, whereas the other parameters have already more than $55 \%$ errors for the 2-h scan.

Another approach would be to set $k_{4}$ to zero to minimize errors in the parameter estimates. Although $k_{4}$ is small, it cannot be neglected as we have shown in earlier work $(2,16)$. Muzi et al. came to a similar conclusion in their mathematic studies (16).

The subdivision into groups G1, G2, and G3 was based on survival-that is, average survival of 3.4, 10.2, and 18.5 mo, respectively. In general, the largest relative change in parameters was seen between baseline and 2 wk of therapy $(\Delta \mathrm{S} 1 \rightarrow \mathrm{S} 2)$, as shown in Figures 3 and 7 . Thereafter, the trend reveals that the changes persist for G3, whereas G1 returns to normal. These changes between baseline and end of therapy monitoring $(\Delta \mathrm{S} 1 \rightarrow \mathrm{S} 3)$ revealed signif-

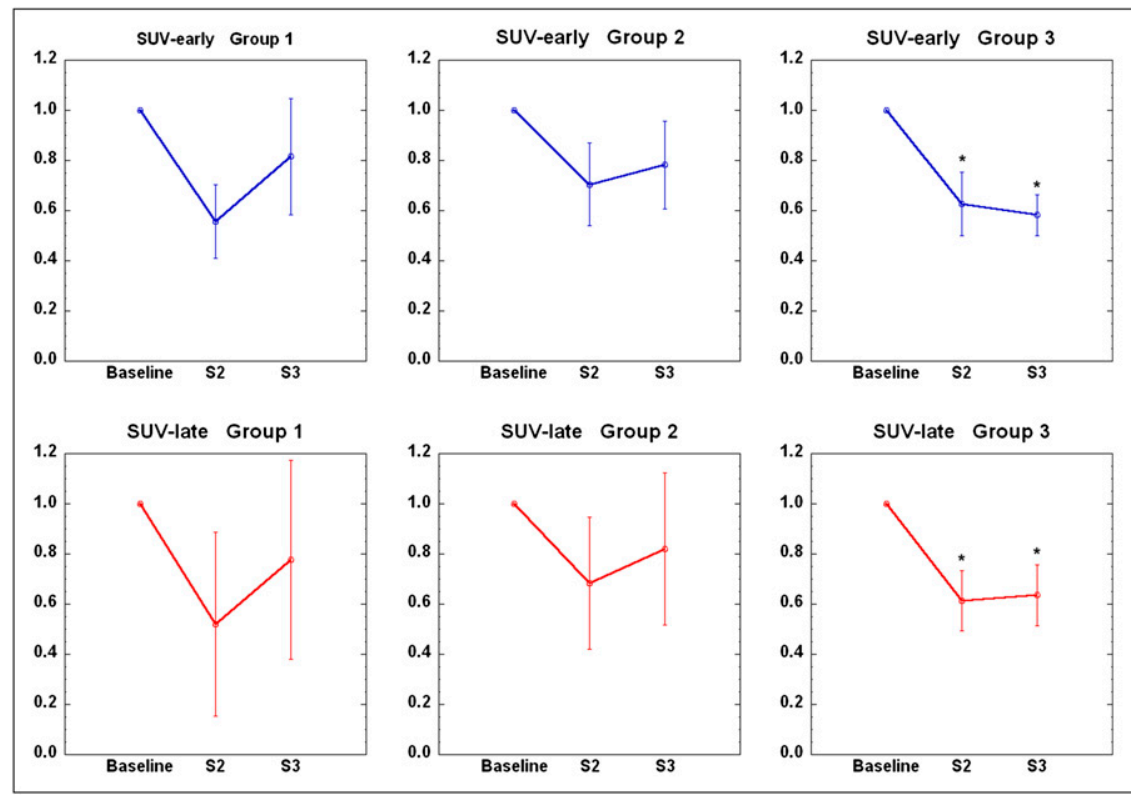

FIGURE 7. Average relative change in ${ }^{18} \mathrm{~F}-\mathrm{FLT}$ uptake SUV $\mathrm{V}_{\text {early }}$ (top, blue) and $\mathrm{SUV}_{\text {late }}$ (bottom, red) from $\mathrm{S} 1 \rightarrow \mathrm{S} 2$ and $\mathrm{S} 1 \rightarrow \mathrm{S} 3$ with method F2-cube. Three groups of patients are shown: G1 (survival, <6 mo), G2 (survival, 6-12 $\mathrm{mo}$ ), and G3 (survival, >12 mo). Results are normalized to baseline study (SUV $=1)$; bars denote SD. Significant differences $(P<0.01)$ are denoted with an asterisk and were found for G3 both for SUV early $_{\text {and }}$ SUV late. 
icant differences for $\mathrm{V}_{\mathrm{b}}$ and $\mathrm{V}_{\mathrm{d}}$, and for $\mathrm{K}_{\mathrm{i}}, \mathrm{SUV}_{\text {early, and }}$ $\mathrm{SUV}_{\text {late}}$, for the analysis methods using a cube (fixed-volume VOI). When thresholding techniques with variable-volume tumor VOIs were used, the number of significant changes decreased for $K_{i}, S U V_{\text {early, and }} S_{U V} V_{\text {late }}$ and mainly for G3, suggesting that a persistently decreased ${ }^{18} \mathrm{~F}$-FLT uptake in the tumor was a predictor for longer survival (Fig. 7).

Other reported studies $(2,16)$ have shown that transport to the cells is fast and primarily determined by perfusion, whereas the phosphorylation reaction $\left(k_{3}\right)$ is the ratelimiting step, determining uptake and retention of ${ }^{18} \mathrm{~F}$ FLT in somatic tissue (16). This finding suggests that simple semiquantitative analysis (SUV) is sufficient for clinical applications, as was shown here (Fig. 6) for the different patient groups (G1, G2, and G3) and time points (S1, S2, and S3). This observation is not supported by the work of the Cologne group, who claims that the association between $\mathrm{K}_{\mathrm{i}}$ and uptake is tumor grade-dependent and does not apply within 1 World Health Organization grade (11). In an earlier study from this group (8), a significant correlation was found between ${ }^{18} \mathrm{~F}$-FLT uptake and both $k_{1}$ and $k_{3}$, suggesting that the ${ }^{18} \mathrm{~F}$-FLT uptake is related to a combination of factors. As shown in Figure 6, we found an excellent correlation between ${ }^{18}$ F-FLT uptake and influx rate $\mathrm{K}_{\mathrm{i}}$ (containing both $k_{1}$ and $k_{3}$ ). Contrary to the studies of the Cologne group, we corrected the input function for metabolites. In addition, we enrolled only patients with recurrent glioma. The correlations between $k_{1}$ and SUV were always high $(r>0.7)$, but between $k_{3}$ and SUV, we found a correlation coefficient of $0.6-0.7$ for the baseline study (S1) only. The later studies S2 and S3 did not show any relationship $(r<0.2)$.

Other investigators have used ${ }^{11} \mathrm{C}$-methionine for evaluating response to therapy. Galldiks et al. (23) reported that ${ }^{11} \mathrm{C}$-methionine was able to measure a response after 3 cycles of temozolomide, offering a method to measure biologic activity. Jacobs et al. (8) compared ${ }^{11} \mathrm{C}$-methionine directly with ${ }^{18} \mathrm{~F}$-FLT and indicated that the 2 tracers have an uptake that is not related. ${ }^{11} \mathrm{C}$-methionine has a high sensitivity and specificity for glioma. These authors suggested that ${ }^{18} \mathrm{~F}$ FLT helps differentiate low- from high-grade tumors and yields complementary information. Ceyssens et al. (24), on the other hand, argue that interindividual ${ }^{11} \mathrm{C}$-methionine uptake does not permit individual grading. They conclude that the clinical use of ${ }^{11} \mathrm{C}$-methionine is limited to recurrence detection, biopsy guidance, and radiation therapy target delineation. In our study, we found significant changes in ${ }^{18} \mathrm{~F}$-FLT uptake and influx rate in a subgroup of long-term survivors after 1 course of therapy.

In the National Cancer Institute trial, ${ }^{18} \mathrm{~F}$-FLT was able to separate recurrence from radionecrosis (12). Parameters $\mathrm{K}_{\mathrm{i}}$ and $k_{3}$ reached statistical significance. These authors emphasized that simple blood-brain barrier breakdown might increase transport without affecting the influx (retention in the salvage pathway). This finding is not consistent with what we reported earlier (5). ${ }^{18}$ F-FLT uptake was measurable in stable patients with recurrent glioma, who had an intact blood-brain barrier, because they had no contrast enhancement on gadolinium-enhanced T1-weighted MRI (4).

Limitations of this study are the small numbers of patients per subgroup. On the other hand, all tumors were recurrent and high-grade, minimizing heterogeneity. Despite the small number of patients, robust effects were found for the relative change of parameters between $S 1$ and $\mathrm{S} 2(\Delta \mathrm{S} 1 \rightarrow \mathrm{S} 2)$ that were statistically significant. If the decreased uptake of ${ }^{18}$ F-FLT persists between S2 and S3, the patient is likely to have a good prognosis and will survive longer (G3 in Fig. 7).

\section{CONCLUSION}

${ }^{18}$ F-FLT kinetics were investigated during treatment of recurrent high-grade brain tumors. The standard 3-compartment model with corrections for $\mathrm{V}_{\mathrm{b}}$, metabolites, and partial volume was used to estimate kinetic parameters. Several analysis methods, including variable versus fixed tumor VOI (defined on factor vs. summed images), were compared. A cube with a fixed volume of $1.2 \mathrm{~cm}^{3}$ defined on the summed images from 45 to $60 \mathrm{~min}$ after ${ }^{18} \mathrm{~F}$-FLT injection yielded the highest number of significant differences between the subgroups. The largest change of kinetic parameters occurred between baseline and $2 \mathrm{wk}$ of treatment. Significant changes were found for $V_{b}, K_{i}, V_{d}$, $\mathrm{SUV}_{\text {early, and }} \mathrm{SUV}_{\text {late }}$ After stratification to overall survival, the patients with the best prognosis showed a change at $2 \mathrm{wk}$ of treatment that persisted at $6 \mathrm{wk}$, whereas the patients with short survival times $(\mathrm{G} 1)$ returned to baseline values at $6 \mathrm{wk}$. High correlations were found between ${ }^{18} \mathrm{~F}$ FLT uptake in tumor (SUV) and influx rate constant $\left(\mathrm{K}_{\mathrm{i}}\right)$, indicating that in clinical practice simple uptake measurements are sufficient for therapy monitoring and predicting short- and long-term survival.

\section{REFERENCES}

1. Shields AF, Grierson JR, Dohmen BM, et al. Imaging proliferation in vivo with [F-18]FLT and positron emission tomography. Nat Med. 1998;4:1334-1336.

2. Shields AF. PET imaging with ${ }^{18} \mathrm{~F}-\mathrm{FLT}$ and thymidine analogs: promise and pitfalls. J Nucl Med. 2003;44:1432-1434.

3. Toyohara J, Waki A, Takamatsu S, Yonekura Y, Magata Y, Fujibayashi Y. Basis of FLT as a cell proliferation marker: comparative uptake studies with $\left[{ }^{3} \mathrm{H}\right]$ thymidine and $\left[{ }^{3} \mathrm{H}\right]$ arabinothymidine, and cell-analysis in 22 asynchronously growing tumor cell lines. Nucl Med Biol. 2002;29:281-287.

4. Chen W, Cloughesy T, Kamdar N, et al. Imaging proliferation in brain tumors with ${ }^{18}$ F-FLT PET: comparison with ${ }^{18}$ F-FDG. J Nucl Med. 2005;46:945-952.

5. Chen W, Delaloye S, Silverman DH, et al. Predicting treatment response of malignant gliomas to bevacizumab and irinotecan by imaging proliferation with $\left[{ }^{18} \mathrm{~F}\right]$ fluorothymidine positron emission tomography: a pilot study. J Clin Oncol. 2007;25:4714-4721.

6. Schiepers C, Chen W, Dahlbom M, Cloughesy T, Hoh CK, Huang SC. ${ }^{18}$ Ffluorothymidine kinetics of malignant brain tumors. Eur J Nucl Med Mol Imaging. 2007;34:1003-1011.

7. Van Laere $\mathrm{K}$, Ceyssens $\mathrm{S}$, Van Calenbergh $\mathrm{F}$, et al. Direct comparison of ${ }^{18} \mathrm{~F}-$ FDG and ${ }^{11} \mathrm{C}$-methionine PET in suspected recurrence of glioma: sensitivity, inter-observer variability and prognostic value. Eur J Nucl Med Mol Imaging. 2005;32:39-51.

8. Jacobs AH, Thomas A, Kracht LW, et al. ${ }^{18} \mathrm{~F}$-fluoro-L-thymidine and ${ }^{11} \mathrm{C}$ methylmethionine as markers of increased transport and proliferation in brain tumors. J Nucl Med. 2005;46:1948-1958. 
9. Popperl G, Kreth FW, Herms J, et al. Analysis of ${ }^{18}$ F-FET PET for grading of recurrent gliomas: is evaluation of uptake kinetics superior to standard methods? J Nucl Med. 2006;47:393-403.

10. Chen W. Clinical applications of PET in brain tumors. J Nucl Med. 2007;48: 1468-1481.

11. Ullrich $\mathrm{R}$, Backes $\mathrm{H}, \mathrm{Li} \mathrm{H}$, et al. Glioma proliferation as assessed by $3^{\prime}$-fluoro3'-deoxy-L-thymidine positron emission tomography in patients with newly diagnosed high-grade glioma. Clin Cancer Res. 2008;14:2049-2055.

12. Spence AM, Muzi M, Link JM, et al. NCI-sponsored trial for the evaluation of safety and preliminary efficacy of $3^{\prime}$-deoxy- $3^{\prime}-\left[{ }^{18} \mathrm{~F}\right]$ fluorothymidine (FLT) as a marker of proliferation in patients with recurrent gliomas: preliminary efficacy studies. Mol Imaging Biol. 2009;11:343-355.

13. Yun M, Oh SJ, Ha HJ, Ryu JS, Moon DH. High radiochemical yield synthesis of $3^{\prime}$-deoxy- $3^{\prime}-\left[{ }^{18} \mathrm{~F}\right]$ fluorothymidine using $\left(5^{\prime}-O\right.$-dimethoxytrityl-2'-deoxy-3'-Onosyl-beta-D-threo pentofuranosyl)thymine and its 3- $N$-BOC-protected analogue as a labeling precursor. Nucl Med Biol. 2003;30:151-157.

14. Schiepers C, Hoh CK, Nuyts J, et al. $1{ }^{11} \mathrm{C}$-acetate kinetics of prostate cancer. J Nucl Med. 2008;49:206-215.

15. Schiepers C, Hoh CK, Dahlbom M, Wu HM, Phelps ME. Factor analysis for delineation of organ structures, creation of in- and output functions, and standardization of multicenter kinetic modeling. Proc SPIE. 1999;3661:1343-1350.

16. Muzi M, Mankoff DA, Grierson JR, Wells JM, Vesselle H, Krohn KA. Kinetic modeling of $3^{\prime}$-deoxy-3'-fluorothymidine in somatic tumors: mathematical studies. J Nucl Med. 2005;46:371-380.
17. Schiepers $\mathrm{C}, \mathrm{Wu} \mathrm{HM}$, Nuyts $\mathrm{J}$, et al. F-18 fluoride PET: is non-invasive quantitation feasible with factor analysis [abstract]? J Nucl Med. 1997;38 (5, suppl):93P.

18. Schiepers C, Hoh CK, Dahlbom M, Wu HM, Phelps ME. Reproducibility of input functions obtained with factor analysis in breast cancer [abstract]. $J$ Nucl Med. 1998;39(5, suppl):165P.

19. Schiepers C, Hoh CK, Seltzer MA, Phelps ME, Dahlbom M. Factor analysis for quantification of ${ }^{11} \mathrm{C}$-acetate PET in primary prostate cancer [abstract]. $\mathrm{J} \mathrm{Nucl}$ Med. 2000;41(5, suppl):100P.

20. Schiepers C, Hoh CK, Nuyts J, Wu HM, Phelps ME, Dahlbom M. Factor analysis in prostate cancer: delineation of organ structures and automatic generation of in- and output functions. IEEE Trans Nucl Sci. 2002;49:23382343.

21. Wu HM, Hoh CK, Choi Y, et al. Factor analysis for extraction of blood timeactivity curves in dynamic FDG-PET studies. J Nucl Med. 1995;36:1714-1722.

22. Schiepers C, Czernin J, Hoh CK, Nuyts J, Phelps ME, Dahlbom M. Factor analysis for automatic determination of myocardial flow in ${ }^{13} \mathrm{~N}$-ammonia PET studies [abstract]. J Nucl Med. 2002;43(5, suppl):52P.

23. Galldiks N, Kracht LW, Burghaus L, et al. Use of ${ }^{11} \mathrm{C}$-methionine PET to monitor the effects of temozolomide chemotherapy in malignant gliomas. Eur $J$ Nucl Med Mol Imaging. 2006;33:516-524.

24. Ceyssens S, Van Laere K, de Groot T, Goffin J, Bormans G, Mortelmans L. $\left[{ }^{11} \mathrm{C}\right]$ methionine PET, histopathology, and survival in primary brain tumors and recurrence. AJNR. 2006;27:1432-1437. 